\title{
$\alpha$-Ketoadipic Aciduria: A Description of a New Metabolic Error in Lysine-Tryptophan Degradation
}

\author{
R. W. WILSON, ${ }^{(31)}$ C. M. WILSON, S. C. GATES, AND J. V. HIGGINS \\ Departments of Natural Science. Zoology, Biochemistry, and Human Development, Michigan State University, \\ East Lansing. Michigan USA
}

\begin{abstract}
Extract
Our studies of a mentally retarded male with extremely elevated levels of $\alpha$-aminoadipic acid and $\alpha$-ketoadipic acid in his urine have led to the description of a new metabolic defect, $\alpha$-ketoadipic aciduria. Analysis of the urine and serum from the patient's family revealed that the patient $(K W)$ had a mentally and physically normal sister $(C W)$ with the same metabolites elevated, but the rest of the family appeared normal.
\end{abstract}

\section{Speculation}

The presence of elevated levels of $\alpha$-ketoadipate in the urine of the reported sibs suggests a reduced level of $\alpha$-ketoadipic acid dehydrogenase. This enzyme has not been purified in humans, but is considered to be $\alpha$-ketoglutaric acid dehydrogenase in several nonhuman animals. We are presenting evidence that would suggest that in humans these may be separate enzymes, or that more than one form of the $\alpha$-ketoglutaric acid dehydrogenase exists.

During the routine testing for amino acid anomalies in Michigan state hospitals for the mentally retarded, an unidentified ninhydrin-positive compound was found in the urine of a 14-year-old male. The unknown was also present in his serum and later identified as $\alpha$-aminoadipic acid ( $\alpha$-AA). Further studies revealed that the urine also contained $\alpha$-ketoadipic acid ( $\alpha$-KA). Neither of these metabolites appeared in measurable amounts using colorimetric methods in the urine or serum of normal control subjects or of other patients (20). Analysis of the patient's family revealed that a mentally normal sister $(C W)$ had the same metabolic pattern.

A search of the literature has revealed that a patient with hyperlysinemia had a slight elevation of $\alpha$-AA but normal levels of $\alpha$-KA (21). In reporting another error in lysine metabolism, saccharopinuria, $\alpha$-AA was reported to be present in the urine (3). Normal levels of $\alpha$-AA have also been reported in a patient with hyperpipecolatemia (7). In all of these cases, the enzymatic error was considered to be at a point between lysine and $\alpha$-AA.

The generally accepted origins of $\alpha$-AA and $\alpha-\mathrm{KA}$ are lysine and lysine/tryptophan, respectively. The transamination of $\alpha$-AA gives rise to $\alpha-\mathrm{KA}$ and requires $\alpha$-ketoglutarate and $\mathrm{B}_{6}$ as cofactors. However, the reductive deamination of $\alpha$-aminomuconic acid, a metabolite of tryptophan, also yields $\alpha$-KA. Subsequently, $\alpha$-KA undergoes an oxidative decarboxylation to yield glutaryl-CoA and $\mathrm{CO}_{2}$. The cofactors which have been implicated in the oxidative decarboxylation of $\alpha$-keto acids have been shown to be: CoASH, NAD, thiamine pyrophosphate, lipoic acid, and FAD.

Scheme 1 is a summary of the proposed steps in the origin and degradation of $\alpha$-AA and $\alpha$-KA.

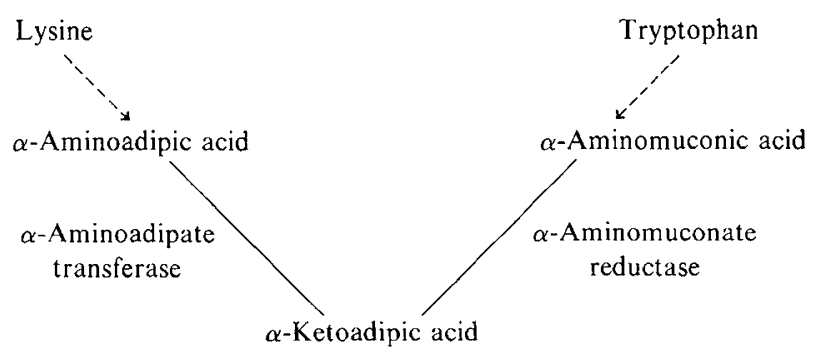

$\alpha$-Ketoadipate dehydrogenase ( $\alpha$-ketoglutarate dehydrogenase?) plus cofactors

Glutaryl-CoA plus $\mathrm{CO}_{2}$

Acetoacetyl-CoA

Scheme 1. Steps in degradation pathway.

MATERIALS AND METHODS

PATIENT

The subject $(K . W)$ is a Caucasian male and was born in July 1960 after an apparently normal pregnancy. His birth weight was $6 \frac{1}{2}$ pounds. His developmental milestones were slow and he has never talked. His hearing was diagnosed to be normal at 34 months. He has always been difficult to feed and prefers dry food. His I.Q. is estimated to be less than 25 , and he is self-abusive. Physically he is very thin but otherwise normal.

\section{FAMILY}

There is no history of mental or physical defects on either side of the family and no consanguinity could be established, although the parents both are of German ancestry. The full and half siblings of $K W$ (Fig. 4) are all mentally and physically normal.

\section{IDENTIFICATION OF $\alpha$-AMINOADIPIC ACID AND $\alpha$-KETOADIPIC} ACID

Descending paper chromatography was employed in the initial studies (solvent systems used are given in Table 1). A Technicon automatic amino acid analyzer was used for evaluating the patients and identifying the unknown compound. The columns were packed with Chromo-beads, type $B$, and the samples were eluted at a rate of $4 \mathrm{ml} / \mathrm{min}$ using sodium citrate buffer, $\mathrm{pH}$ 2.875-5.00. Authentic samples of $\alpha$-AA and $\alpha$-KA were purchased from the Sigma Chemical Company. Dinitrophenyl hydrazone derivatives of the $\alpha$-ketoacids were prepared by established procedures (15). 


\section{GAS CHROMATOGRAPHY-MASS SPECTROMETRY}

Organic acids were separated from $1 \mathrm{ml}$ urine using a modification of an established procedure (4). Oximes of keto acids were formed at $\mathrm{pH} 10$ with added hydroxylamine hydrochloride $(75$ $\mathrm{mg}$ ), by heating the mixture for $20 \mathrm{~min}$ at $82^{\circ}(13)$. The urine was cooled, brought to $\mathrm{pH} 7.0$ with dilute hydrochloric acid, and placed in a small DEAE-Sephadex (acetate form) column. Organic bases and neutral compounds were eluted with water and discarded, and molecules with a net negative charge at $\mathrm{pH} 7$ were eluted with 1.5 $M$ pyridinium acetate. The sample was frozen and lyophilized at $0^{\circ}$ and 0.5 Torr for 5-6 hours, or until dry. If necessary, thiophene-free benzene (Aldrich (22)) was added and lyophilized to remove final traces of water from the sample. Samples were transferred to conical centrifuge tubes in methanol and the solvent was removed with nitrogen gas; the residues were dissolved in 300 $\mu \mathrm{l}$ bis-trimethylsilyl trifluoracetamide which contained $1 \%$ by volume of trimethylchlorosilane (Pierce (23)). After heating for 1 $\mathrm{hr}$ at $82^{\circ}$, the sample was stored in sealed silanized glass capillaries at $4^{\circ}$. Trimethylsilyl (TMS) oxime of a reference sample of $\alpha$-KA (Sigma (24)) was prepared in pyridine using standard methods (13), and the TMS derivative of $\alpha$-AA by dissolving the reference sample (Sigma) in pyridine-bis-trimethylsilyl trifluoracetamide, $1: 1$, and heating for $1 \mathrm{hr}$ at $82^{\circ}$.

Samples $(2 \mu \mathrm{l})$ were chromatographed on a Varian 2100 gas chromatograph (Varian Aerograph (25)) equipped with flame ionization detectors. Glass paperclip-shaped columns $(2 \mathrm{~mm}$ i.d. $\times$ $3.8 \mathrm{~m}$ ) were packed with either $3 \%$ SE-30 or $3 \%$ OV-17 on $80 / 100$ mesh Supelcoport (Supelco (26)) and programmed from $120-270^{\circ}$

Table 1. Migration values for urine unknowns, $\alpha$-aminoadipic acid $(\alpha-A A)$ and $\alpha$-ketoadipic acid $(\alpha-K A)$

\begin{tabular}{lcccccc}
\hline & B-A $^{1}$ & P-E-A & Py-B & B-E-A & tA-E & E time \\
\hline$\alpha$-AA & 0.38 & 0.12 & 0.21 & & & $6 \mathrm{hr} 52 \mathrm{~min}$ \\
Unknown & 0.38 & 0.12 & 0.21 & & & $6 \mathrm{hr} 52 \mathrm{~min}$ \\
DNP- $\alpha$-KA & & & & 0.52 & 0.030 & \\
DNP-unknown & & & 0.52 & 0.30 & \\
\hline
\end{tabular}

${ }^{1}$ BA: butanol-acetic acid-water (12:3:5); P-E-A: phenol-ethanolammonia (15:4:1); Py-B: pyridine-butanol-water (1:1:1); B-E-A: butanolethanol-ammonia (13:2:5); tA-E: tert-amyl alcohol-ethanol-water (5:1:4, upper layer); E time: time for elution from amino acid analyzer; DNP: dinitrophenyl. at $4^{\circ} / \mathrm{min}$. Flow rates were 30,24 , and $300 \mathrm{ml} / \mathrm{min}$ for helium, hydrogen, and air, respectively.

Coiled glass columns with the same packings and dimensions were used in an LKB-9000 combined gas chromatograph-mass spectrometer. The magnetic field of the mass spectrometer was repetitively scanned at 6-sec intervals from $m / e$ 50-500 during the entire gas chromatographic temperature-programmed run. Data were acquired and processed on a PDP-8/e computer (Digital Equipment (27)) with 8,000 words of core memory and a 1.6 million word disk, utilizing an updated version of an earlier data system (18).

\section{RESULTS}

The initial urine screens for amino acid anomalies were done on nearly 14,000 patients using standard chromatographic techniques with butanol-acetic acid-water $(12: 5: 3)$ as the solvent. In the urine of the patient $(K W)$ a very pronounced spot which corresponded to alanine was noticed. When a 24 -hr urine sample was passed through the amino acid analyzer it was revealed that the alanine level for $K W$ was normal, but that a unique peak was present. This unidentified compound was subsequently shown to be $\alpha$-AA. The results in Table 1 show that the $R_{F}$ values for the unknown and commercial $\alpha$-AA were the same for the three solvents used. Authentic $\alpha$-AA also migrated as a single spot when cochromatographed with the unknown. Additional studies revealed the presence of $\alpha$-AA in the serum of the patient (Tables 2 and 3).

Table 2. Quantitative amounts of $\alpha$-aminoadipic acid $(\alpha-A A)$ and $\alpha$-ketoadipic acid $(\alpha-K A)$ present in urine and serum

\begin{tabular}{|c|c|c|c|c|}
\hline & \multicolumn{2}{|c|}{ Urine } & \multicolumn{2}{|c|}{ Serum } \\
\hline & $\begin{array}{c}\alpha-\mathrm{AA}, \\
\mu \mathrm{mol} / 24 \mathrm{hr}^{1}\end{array}$ & $\begin{array}{c}\alpha-\mathrm{KA} \\
\mu \mathrm{mol} / 100 \mathrm{ml}^{2}\end{array}$ & $\begin{array}{c}\alpha-\mathrm{AA} \\
\mu \mathrm{mol} / \mathrm{ml}\end{array}$ & $\alpha-\mathrm{KA}$ \\
\hline$K W$ & 1,480 & 62 & 0.034 & Trace \\
\hline$C W$ & 1,500 & 245 & 0.057 & Trace \\
\hline Controls $^{3}$ & 0 -Trac & Undetectable & 0 -Trace & Trace \\
\hline
\end{tabular}

${ }^{1}$ Determined on amino acid analyzer.

${ }^{2}$ Determined as dinitrophenyl hydrazones.

${ }^{3}$ Controls include members of the patient's family, the investigators, several age-matched children, and several hundred patients from Michigan state institutions.

Table 3. Quantitative analysis of urine and serum amino acids ${ }^{1}$

\begin{tabular}{|c|c|c|c|c|c|c|}
\hline \multirow[b]{2}{*}{ Amino acids } & \multicolumn{3}{|c|}{ Urine $(\mu \mathrm{M} / 24 \mathrm{hr})$} & \multicolumn{3}{|c|}{$\operatorname{Serum}(\mu \mathrm{M} / \mathrm{min})$} \\
\hline & $K W$ & $C W$ & Normal range & $K W$ & $C W$ & Normal range \\
\hline Aspartic acid & & & & 0.042 & 0.052 & $0-0.10$ \\
\hline Threonine & 673 & 284 & $187-1,370$ & 0.434 & 0.333 & $0.348-0.870$ \\
\hline Serine & 342 & 223 & $110-871$ & 0.155 & 0.150 & $0.122-0.252$ \\
\hline Glutamic & & & & 0.136 & 0.197 & $0.058-0.397$ \\
\hline$\alpha$-Aminoadipic acid & 1,480 & 1,500 & Trace & 0.034 & 0.058 & $0-$ Trace \\
\hline Glycine & 550 & 800 & $785-3,918$ & 0.258 & 0.253 & $0.211-0.438$ \\
\hline Alanine & 176 & 240 & $88-541$ & 0.303 & 0.453 & $0.268-0.701$ \\
\hline Valine & & & & 0.173 & 0.300 & $0.139-0.315$ \\
\hline Isoleucine & & & & 0.041 & 0.092 & $0.032-0.102$ \\
\hline Leucine & & & & 0.088 & 0.185 & $0.047-0.135$ \\
\hline Tryosine & 97 & 163 & Trace-229 & 0.052 & 0.085 & $0.047-0.135$ \\
\hline Phenylalanine & Trace & 45 & $0-262$ & 0.237 & 0.079 & $0.054-0.133$ \\
\hline Ornithine & & & & 0.060 & 0.094 & $0.045-0.186$ \\
\hline Lysine & 70 & 542 & $21-1,046$ & 0.201 & 0.206 & $0.128-0.320$ \\
\hline Histidine & 703 & 1,100 & $470-2,843$ & 0.072 & 0.113 & $0.096-0.207$ \\
\hline
\end{tabular}

- Amino acids given in the order in which they are eluted from column. 
After identifying the unknown in the patient's urine, it was decided to look for additional accumulation products of lysine metabolism. Because the transamination of $\alpha$-AA yields $\alpha$-KA, the ketoacids present in the urine of $K W$ were determined after converting them to their dinitrophenyl hydrazone derivatives. The results showed that in addition to the derivatives of $\alpha$-ketoglutarate and pyruvate, found in the control subjects, $K W$ again had a unique compound. The migration values shown in Table 1 indicated that $\alpha$-KA was present.

Urine samples from $K W, C W$, and a 7-year-old control subject with no known metabolic defects were analyzed using both gas chromatography and mass spectrometry. Two major peaks in the urines of both $K W$ and $C W$ were found to be eluted at temperatures apparently identical with those of the derivatives of $\alpha-\mathrm{KA}$ and $\alpha$-AA on both OV-17 (Fig. 1) and SE-30; the two compounds were incompletely resolved from one another on the latter column. When spectra were collected during a similar separation on an OV-17 column in the gas chromatograph-mass spectrometer, these same two peaks were found to have spectra essentially identical with those of the pure standards (Figs. 2 and 3). Other major peaks
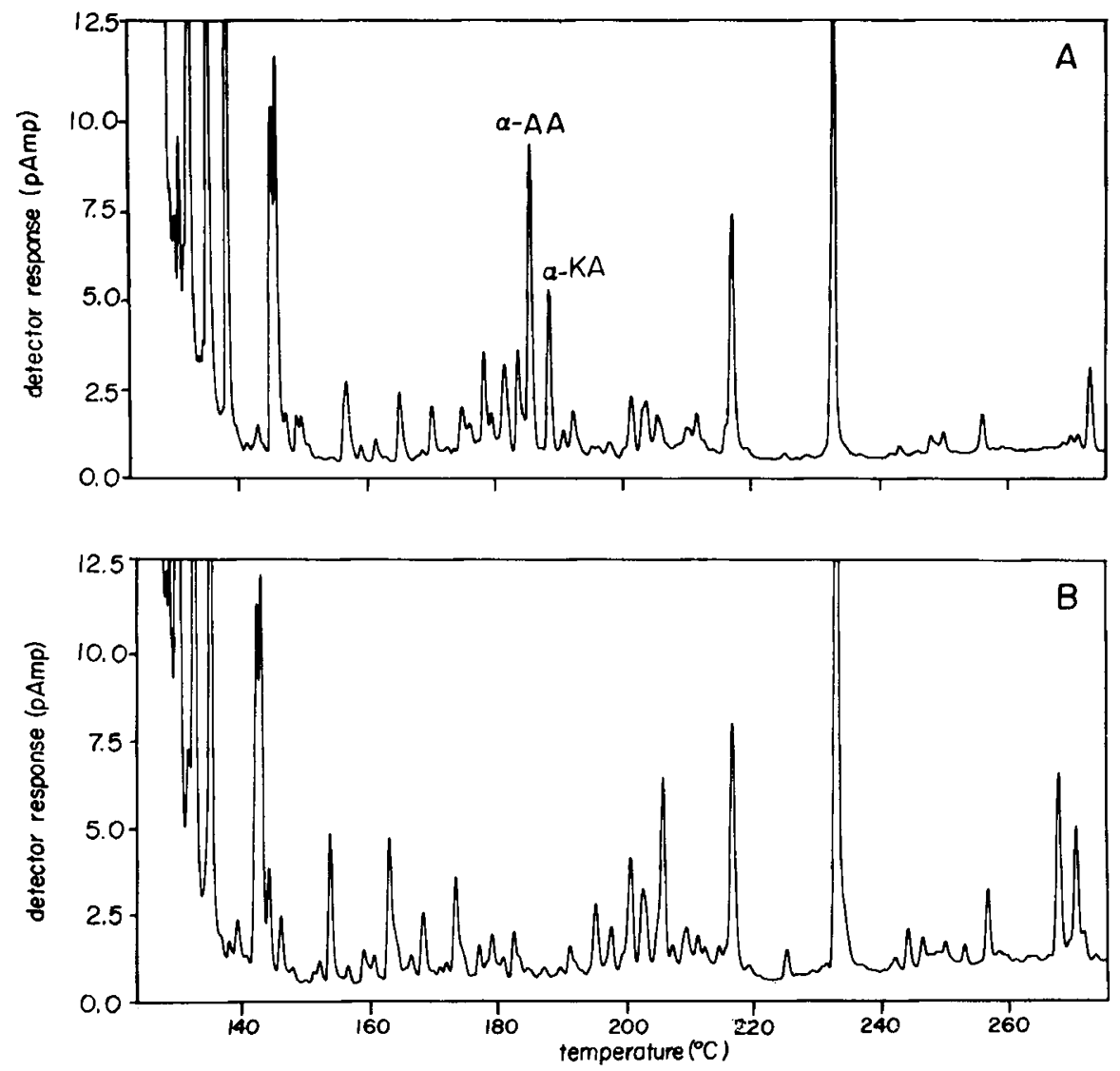

Fig. 1. Gas chromatography of urines prepared by an anion exchange procedure. Both the urine of the patient $(K W)$ (chromatogram A) and that of the control subject $(B)$ show similar peaks, except for the two peaks identified as $\alpha$-aminoadipic acid $(\alpha-A A)$ and $\alpha$-ketoadipic acid $(\alpha-K A)$. Both chromatograms represent injections equivalent to $67 \mu \mathrm{l}$ urine. However, $A$ represents $2.6 \mu \mathrm{g}$ creatinine compared with $6.6 \mu \mathrm{g}$ creatinine equivalent injected for the control subject.
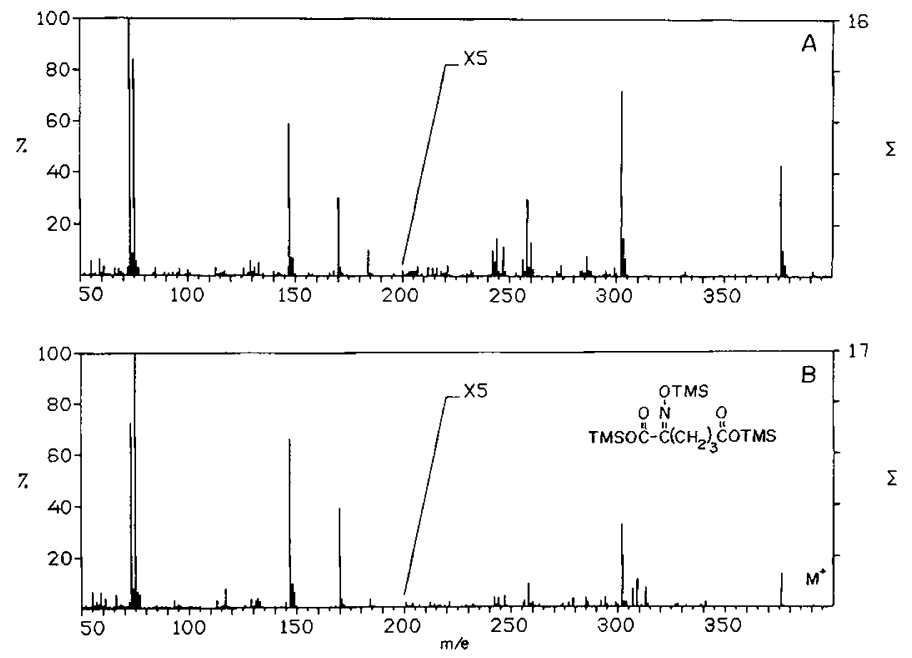

Fig. 2. A: Mass spectrum of the peak lbeled $\alpha$-ketoadipic acid $(\alpha-K A)$ in Figure 1; $B$ : the oxime trimethylsilyl $(T M S)$ derivative of reference $\alpha-K A$. The olecular ion of the reference compound is observed at $m / e 391$. 

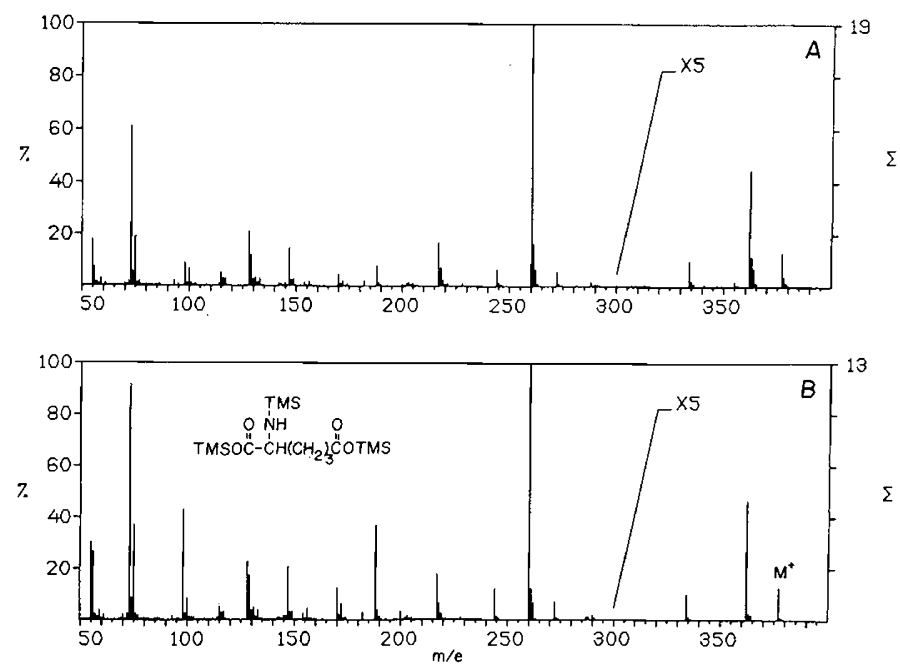

Fig. 3. Comparison of the mass spectrum of peak $\alpha$-aminoadipic acid $(\alpha-A A)$ in Figure $1(A)$ to that of the pure trimethylsilyl $(T M S) \alpha-A A(B)$. The molecular ion is observed at $m / e 377$.
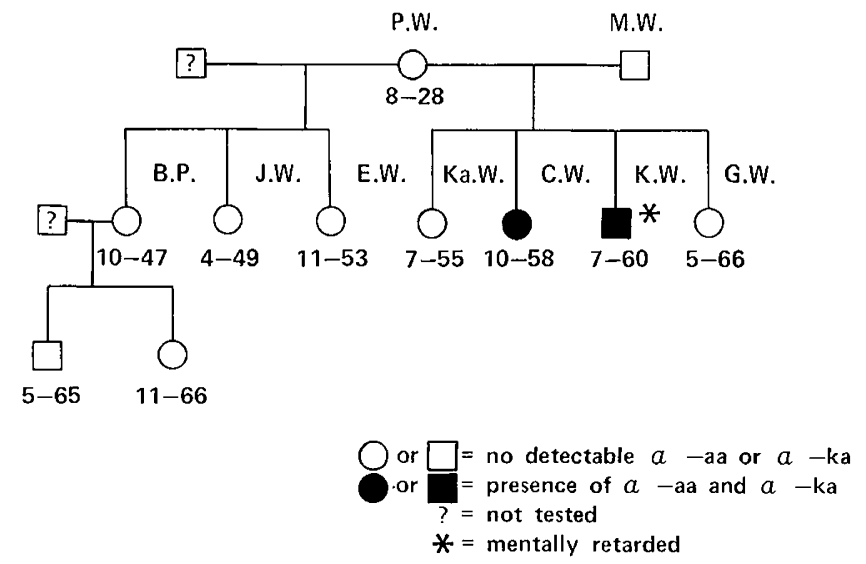

Fig. 4. Pedigree of family with $\alpha$-aminoadipic and $\alpha$-ketoadipic acid anomaly.

in the $K W$ urine sample, particularly those eluting close to the peaks of interest, were examined and found to be similar to peaks at similar retention times in the control subject's urine. Also using mass spectrometer techniques, samples of urine from $K W$ and $C W$ were analyzed by Dr. Stephen Goodman (Department of Pediatrics, University of Colorado Medical Center, Denver). He too confirmed the presence of $\alpha-\mathrm{KA}$ in the samples.

\section{STUDIES OF $K W$ S FAMILY}

Repeated serum and 24-hr urine samples have been collected from the patient's family and tested colorimetrically as above. These studies revealed that one mentally normal sister $(C W)$ had a similar metabolic pattern. The pedigree (Fig. 4) suggests that the defect behaves as an autosomal recessive.

\section{QUANTITATION OF $\alpha$-AA, $\alpha$-KA AND OTHER METABOLITES}

The amino acid levels in the blood and urine of $K W$ and $C W$ are presented in Table 3. In both cases, the $\alpha$-AA levels greatly exceed control levels. Additionally, $K W$ had high serum levels of phenylalanine but the amount is below that characteristic of phenylketonuria. The other family members had normal amino acid levels.

The $\alpha$-KA levels were determined by treating known amounts of urine and $\alpha-\mathrm{KA}$ in identical fashion and quantitatively recovering the products. After chromatography, the dinitrophenyl-hydrazone derivatives were eluted in constant volumes of sodium hydroxide and determined colorimetrically at $430 \mathrm{~nm}$. The results are shown in Table 2. The quantitative analysis of $\alpha$-ketoglutarate and pyruvate revealed that the affected sibs were in the normal range.

When age-matched control urine was examined by gas chromatography-mass spectrometry, it was found to contain $\alpha$-AA; estimates based on relative sizes of ion peaks compared with those in the $K W$ urine sample would suggest that $\alpha$ - $\mathrm{AA}$ is present in the control urine at approximately $3-4 \%$ of the level in a similar volume of the $K W$ urine. No peaks definitely associated with $\alpha$-KA could be detected in the control subject's urine. Hence, since the lower limit of detectability with the current system is about 3 $\mu \mathrm{g}$ of $\alpha$-KA ml urine, the urine samples for the two sibs appear to contain levels which are at least 30-50 times higher than normal for $\alpha$-KA. When based on a 24 -hr output or on creatinine levels (Table 1 and Fig. 1), the comparison is even more striking.

To provide further support for the values obtained from the colorimetric assay, the levels of $\alpha$-KA and $\alpha$-AA in the urine of $K W$ and $C W$ were assessed by adding known amounts of these compounds to the control subject's urine and then completing the usual ion exchange procedure (Materials and Methods). Peak areas were used to calculate the approximate amount of each compound present in both urines; the values so obtained agreed with those calculated utilizing the colorimetric assay.

\section{DISCUSSION}

Our studies of a mentally retarded male with extremely elevated levels of $\alpha$-aminoadipic acid and $\alpha$-ketoadipic acid in his urine have led to the description of a new metabolic defect, $\alpha$-ketoadipic aciduria. The $\alpha$-AA, which is also present in his serum, probably arises from lysine catabolism, whereas the $\alpha$-KA could originate from either lysine or tryptophan. Analysis of the urine and serum samples from the patient's family revealed that the patient $(K W)$ had a mentally and physically normal sister $(C W)$ with the same metabolites elevated, but that the rest of the family appeared normal. The fact that repeated samples have been tested, and the different environments of $K W$ and $C W$ point toward a genetic etiology of this condition.

A logical cause for the accumulation of the $\alpha$-KA would be a diminished $\alpha$-KA dehydrogenase activity. Curiously, several reports on non-human mammals have provided evidence which indicated that $\alpha$-ketoglutarate $(\alpha-K G)$ dehydrogenase is the enzyme responsible for the oxidative decarboxylation of $\alpha-\operatorname{KA}(9,12$, 16). If the same is true in humans, $K W$ and $C W$ should also have elevated levels of $\alpha$-KG. Since their $\alpha-K G$ levels were in the normal range, there is the possibility that $\alpha-\mathrm{KA}$ and $\alpha-\mathrm{KG}$ dehydrogenase are separate proteins, or that the defect affects the active sites differently.

In a situation relevant to the case reported here, Goodman et al. 
(8) have reported mentally retarded sibs with glutaric aciduria. Their studies showed a glutaryl-CoA dehydrogenase deficiency in the peripheral leukocytes of the children as compared with control subjects. The glutaryl-CoA dehydrogenase is the next step distal to the $\alpha$-ketoadipate dehydrogenase. In a personal communication with Goodman, it was learned that neither $\alpha$-aminoadipate nor $\alpha$-ketoadipate was elevated in his patients. Taken together these observations support our speculation that the $\alpha$-ketoadipic aciduria is caused by a block at the level of $\alpha$-ketoadipate dehydrogenase. The immediate precursor of $\alpha$-AA is $\alpha$-AA semialdehyde, but we could not detect it in the urine of the affected sibs. Likewise, we were unable to detect any abnormalities in the intermediates of the tryptophan pathway. Particular emphasis was placed on picolinic acid, since $\alpha$-aminomuconic is highly unstable (16). However, a substance presumed to be glutaric acid has been shown by mass spectrometry to be present in the urine of $K W$ and $C W$. The significance of this finding is as yet unclear.

An error at the point of $\alpha$-KA dehydrogenase would be similar to the one(s) in branched chain ketoaciduria (maple syrup urine disease) (5), as well as a recently described pyruvate decarboxylase deficiency (1). That is, they all involve the oxidative decarboxylation of $\alpha$-ketoacids and require the same cofactors. Because various investigations have shown regulatory effects of one ketoacid on the metabolism of one or more of the others, it is of interest to see if $\alpha$-ketoadipate will exert any such effects. For example, in bovine mitochondria, it was found that ketoisocaproate, ketomethylvalerate, and ketoisovalerate not only showed mutually inhibitory effects on the decarboxylation of one another, but also inhibited pyruvate and $\alpha$-ketoglutarate dehydrogenases $(10,11)$. Similarly, ketoisocaproate has been shown to inhibit the oxidative decarboxylation of pyruvate and $\alpha$-ketoglutarate in rat liver slices (2). What, if any, regulatory effect $\alpha$-KA has on these procedures remains to be investigated.

\section{SUMMARY}

This report describes a mentally retarded male and his mentally normal sister who have elevated levels of $\alpha$-aminoadipic and $\alpha$-ketoadipic acids in their urine. The former metabolite is also present in their serum. These findings suggest a new inborn error in the catabolism of lysine and tryptophan.

\section{ADDENDUM}

A second case involving elevated levels of $\alpha$-AA and $\alpha$-KA in a $1 \frac{1}{2}$-year-old female with psychomotor retardation has been reported (17). According to the authors, $\alpha$-AA excretion was correlated with lysine uptake. In a companion paper evidence was presented to indicate that this condition was probably caused by a defect in the $\alpha$-ketoadipate dehydrogenase (19). Their evidence was based on reduction of $\mathrm{CO}_{2}$ evolution from $\alpha-\mathrm{KA}$ in fibroblasts of the patient.

A family in which sibs have high amounts of $\alpha$-AA but no increase in $\alpha$-KA has also been described (6). In this case, the $\alpha$-AA level was increased by oral doses of lysine in both the retarded male and his normal brother.

Another case of $\alpha$-aminoadipic aciduria has been reported in a 6-year-old oligophrenic male (14). No mention was made of $\alpha$-KA.

\section{REFERENCES AND NOTES}

1. Blass, J. P., Avigan, J., and Uhlendorf, B. W.: A defect in pyruvate decarboxylase in a child with an intermittent movement disorder. J. Clin. Invest., 49: 423 (1970).

2. Bowden, J. A., Brestel, E. P., Cope, W. T., McArthur, C. L., Westfall, D. N., and Fried, M.: $\alpha$-Ketoisocaproic acid inhibition of pyruvate and $\alpha$-ketoglutarate oxidative decarboxylation in rat liver slices. Biochem. Med., 4: 69 (1970).

3. Carson, N. A. J., Scally, B. G., Neill, D. W., and Carre, I. J.: Saccharopinuria: A new inborn error of lysine metabolism. Nature, 218: 679 (1968).

4. Chalmers, R. A., and Watts, R. W. E.: The quantitative extraction and gas-liquid chromatographic determination of organic acids in urine. Analyst, 97: 958 (1972).

5. Dancis, J., and Levitz, M.: Abnormalities of branched-chain amino acids metabolism thypervalinemia, branched-chained ketonuria (maple syrup urine disease) isovaleric acidemia] In: J. B. Stanbury: The Metabolic Basis of Inherited Disease, p. 426, (McGraw-Hill Book Co., New York, 1972).

6. Fischer, M. H., Gerritsen, T., and Opitz, J. M.: $\alpha$-Aminoadipic. aciduria, a non-deleterious inborn metabolic defect. Humangenetik, 24: 265 (1974).

7. Gatfield, P. D., Taller, E., Hinton, G. C., Wallace, A. C., Abdelnour, G. M., and Haust, M. D.: Hyperpipecolatemia: A new metabolic disorder associated with neuropathy and hepatomegaly. Can. Med. Ass. J., 99: 1215 (1968).

8. Goodman, S. I., Markey, S. P., Moe, P. G., Miles, B. S., and Teng, C. C.: Glutaric aciduria: A "new" disorder of amino acid metabolism. Biochem. Med., 12:12 (1975)

9. Hirashima, M., Hayakawa, T., and Koike, M.: Mammalian $\alpha$-ketoacid dehydrogenase complexes. II. J. Biol. Chem., 242: 902 (1967).

10. Johnson, W. A., and Connelly, J. L.: Cellular localization and characterization of bovine liver branched-chain $\alpha$-ketoacid dehydrogenase. Biochemistry, 11: 1967 (1972).

11. Johnson, W. A., and Connelly, J. L.: Studies on the mutual influences of substrates on bovine $\alpha$-ketoacid metabolism. Biochemistry, 11: 2416 (1972).

12. Kanzaki, T., Hayakawa, T., Hamada, M., Fukuyoshi, Y., and Koike, M.: Mammalian $\alpha$-ketoacid dehydrogenase complexes. IV. J. Biol. Chem., 244: 1183 (1969).

13. Lancaster, S., Lamm, P., Scriver, C. R., Tjoa, S. S., and Mamer, O. A.: Quantitative analysis of branched-chain $\alpha$-ketoacids as their trimethylsilylated oximes. Clin. Chim. Acta, 48: 279 (1973).

14. Lormans, S., and Lowenthal, A.: Aminoadipic aciduria in an oligophrenic child. Clin. Chim. Acta, 57: 97 (1974).

15. Menkes, J. H.: Pattern of urinary $\alpha$-ketoacids in various neurological diseases. Amer. J. Dis. Chi'd., 99: 500 (1960).

16. Nishizuka, Y., Ichiyama, A., and Hayaishi, O.: Metabolism of the benzene ring of tryptophan (mammals). V. $\alpha$-Ketoadipate dehydrogenase. Methods Enzymol. 17a: 487 (1970).

17. Przyrembel, H., Bachmann, D., Lombeck, I., Becker, K., Wendel, U., Wadman, S. K., and Bremer, H. J.: Alpha-ketoadipic aciduria, a new inborn error of lysine metabolism: biochemical studies. Clin. Chim. Acta, 58: 257 (1975).

18. Sweeley, C. C., Young, N. D., Holland, J. F., and Gates, S. C.: Rapid computerized identification of compounds in complex biological mixtures by gas chromatography-mass spectrometry. J. Chromatog., 99: 507 (1974).

19. Wendel, U., Rudiger, H. W., Przyrembel, H., and Bremer, H. J.: Alphaketoadipic aciduria: degradation studies with fibroblasts. Clin. Chim. Acta, 58: 271 (1975). •

20. Wilson, C., Wilson, R. W., and Higgins, J. V.: $\alpha$-Ketoadipic aciduria: A new metabolic error in lysine/tryptophan metabolism. 4th International Conference on Birth Defects, No. 297, p. 37 (Excerpta Medica, Amsterdam, 1973).

21. Woody, N. C.: Hyperlysinemia. Amer. J. Dis. Child., 108: 543 (1964).

22. Aldrich Chemical Co., Inc., Milwaukee, Wisc.

23. Pierce Chemical Co., Rockford, Ill.

24. Sigma Chemical Co., St. Louis, Mo.

25. Varian Associates, Palo Alto, Calif.

26. Supelco Inc., Bellefonte, $\mathrm{Pa}$.

27. Digital Equipment Corp., Maynard, Mass.

28. Our grateful appreciation is extended to Mr. Richard Rutz for technical assistance.

29. This work was supported in part by the National Foundation-March of Dimes.

30. We are indebted to Dr. C. C. Sweeley (Department of Biochemistry, Michigan State University) and Dr. S. Goodman (Department of Pediatrics, University of Colorado Medical Center, Denver) for their assistance in mass spectrometer analysis.

31. Requests for reprints should be addressed to R. W. Wilson, Ph.D., Department of Human Development, Michigan State University, East Lansing, Michigan 48824 (USA).

32. Accepted for publication February 28, 1975. 